\title{
Efectividad del programa de atención domiciliaria en el cuidado de úlceras por presión
}

\section{Effectiveness of the domiciliary care program in the care of ulcers by pressure}

Yohanna Ivette Franco Valdez ${ }^{1}$, Susan Haydee Gonzales Saldaña ${ }^{1}$

\section{RESUMEN}

Objetivo: Analizar las evidencias sobre la efectividad del programa de atención domiciliaria en el cuidado de úlceras por presión. Materiales y Métodos: Revisión Sistemática observacional y retrospectivo, la búsqueda se ha restringido a artículos con texto completo, y los artículos seleccionados se sometieron a una lectura crítica, utilizando la evaluación de GRADE para identificar su grado de evidencia. Resultados: En la selección definitiva se eligieron 10 artículos, encontramos con un 30\% encontramos a Brasil, $20 \%$ corresponden a España y Chile, mientras que con un $10 \%$ encontramos a, Argentina, Costa rica y México respectivamente. Han sido estudiados en su mayoría las Revisiones sistemáticas, con un 30\%, un $10 \%$ de Meta análisis y $10 \%$ estudio clínico experimental, principalmente en los países de España, Brasil y Costa rica. Entre los estudios cuantitativos descriptivos encontramos a los países de Brasil y Chile, con un 20\%, con un $10 \%$ tenemos a estudios cuantitativo transversales perteneciente a Brasil, con un $10 \%$ tenemos el estudio de cohorte perteneciente a México y un descriptivo observacional perteneciente a España. Donde del total de artículos analizados el 100\% afirma que los cuidados en ulceras por presión son efectivos en atención domiciliaria. Conclusión: Se evidenció en las investigaciones revisadas que existe efectividad de la atención domiciliaria en el cuidado de úlceras por presión. Se mostró efectividad y calidad en gestión domiciliaria y rehabilitación oportuna de las personas con Úlceras por Presión, aportando al desarrollo y visibilidad disciplinar.

Palabras claves: efectividad, atención domiciliaria, cuidado, ulceras por presión.

\begin{abstract}
Objective: To analyze the evidence on the effectiveness of the home care program in the care of pressure ulcers. Materials and Methods: Observational and retrospective systematic review, the search has been restricted to articles with full text, and the selected articles were subjected to a critical reading, using the evaluation of GRADE to identify its degree of evidence. Results: In the final selection, 10 articles were selected, 30\% found Brazil, 20\% correspond to Spain and Chile, while 10\% found Argentina, Costa Rica and Mexico respectively. Systematic reviews have been studied, with 30\%, 10\% Meta analysis and 10\% experimental clinical study, mainly in the countries of Spain, Brazil and Costa Rica. Among the descriptive quantitative studies we find the countries of Brazil and Chile, with $20 \%$, with $10 \%$ have cross-sectional quantitative studies pertaining to Brazil, with $10 \%$ have the cohort study pertaining to Mexico and an observational descriptive belonging to Spain. Where from the total of articles analyzed $100 \%$ affirm that care in pressure ulcers are effective in home care. Conclusion: It was evidenced in the investigations reviewed that there is effectiveness of home care in the care of pressure ulcers. It showed effectiveness and quality in home management and timely rehabilitation of people with Pressure Ulcers, contributing to the development and disciplinary visibility.
\end{abstract}

Key words: effectiveness, home care, care, pressure ulcers.

${ }^{1}$ Universidad Norbert Wiener. Escuela Profesional de Enfermería. Lima - Perú.

\section{INTRODUCCIÓN}

Ante los cambios demográficos y epidemiológicos que se vienen produciendo a nivel mundial y de manera particular en América
Latina y el Caribe, es indispensable la preparación para responder a las demandas de la creciente población adulta mayor que representa un $12 \%$ de la población mundial (1). 
El envejecimiento humano es un fenómeno universal inevitable y de gran trascendencia debido a las repercusiones sanitarias, económicas y sociales.

Las úlceras por presión, son un problema común a muchos países del mundo, en todos los niveles de atención de la salud, que afecta a personas de todas las edades y que resulta en altos costos económicos, el consumo de recursos y también el sufrimiento individual y familiar (5).

Según reportes a nivel mundial, la población adulta mayor está aumentando considerablemente debido al incremento de la expectativa de vida, disminución de tasas de natalidad y mortalidad, entre otros factores.

Según el acuerdo de Kiev la OMS (1979) considera adultos mayores a los de más de 60 años para los que viven en los países en vías de desarrollo y de 65 años a los que viven en países desarrollados.

La OPS, ajustó la edad de 65 y más para considerarlo como adulto mayor. Para hacer el análisis de la población por estructura de edad en el Perú el Ministerio de Salud (3) presenta el programa de atención integral de salud donde considera al adulto mayor desde los 65 años a más.

En el Perú la población adulta mayor representa el 9,1\% del total de la población, siendo la proyección estimada del $12.5 \%$ para el año 2025 (4).

Ante esta situación, los requerimientos de los servicios sanitarios también se ven incrementados, tal como lo demuestran las estadísticas del Seguro Social de Salud (EsSalud), institución peruana que ha experimentado un progresivo aumento en la atención de las personas adultas mayores, llegando a un $13.2 \%$ del total de atenciones, un $26.4 \%$ de los egresos hospitalarios a nivel nacional y en $32.3 \%$ del total de egresos en Lima (5).

En relación a las causas de hospitalización en adultos mayores, la primera causa son las infecciones: neumonía, infección del tracto urinario y sepsis y accidente cerebrovascular (6). Estos diagnósticos médicos están fuertemente asociados al desarrollo de úlceras por presión en el adulto mayor, por el impacto que tienen estas dos enfermedades en el estado general y en la movilidad de este grupo etáreo. (7), menciona que las úlceras por presión son uno de los síndromes geriátricos que ocasionan mayor inversión socioeconómica y sanitaria. Los efectos de las úlceras por presión radican en una estancia hospitalaria cinco veces mayor que en pacientes sin esta condición, un incremento en la mortalidad, un aumento significativo de complicaciones infecciosas y comorbilidad, y un incremento en los costos de manejo, estimándose en un costo de 489 dólares por úlcera, con un tiempo medio de tratamiento de 116 días (4).

Este problema no es exclusivo de las personas adultas mayores porque puede suceder a cualquier edad, pero los adultos mayores son los que están en mayor riesgo debido a la inmovilización por la declinación biológica normal, la mayor morbilidad, la mayor susceptibilidad a enfermarse, especialmente, por patologías de resolución retardada que reducen aún más el margen de defensa y autonomía (6).

Se dice que, aunque una persona de cualquier edad desarrolla úlceras por presión si no cambia de posición con frecuencia, en los adultos mayores incrementan este riesgo por cambios de la piel relacionados a la edad, incluyendo pérdida de humedad, de grasa subcutánea e incremento en la fragilidad (8).

Las úlceras por presión constituyen un serio problema que afecta principalmente a las personas que permanecen en cama durante largos periodos de tiempo (9), como consecuencia de traumas o enfermedades; afecta la capacidad funcional del adulto mayor, aumentando considerablemente la morbimortalidad de las personas afectadas (10).

En la actualidad a nivel mundial, se encuentran pocos registros sobre la problemática de las úlceras por presión y la prevención de las mismas. Estudios realizados en Europa, Sudáfrica y Canadá permiten establecer que la prevalencia de las personas con úlceras por presión en el medio hospitalario puede llegar hasta un $45 \%$ en los enfermos crónicos y por encima del $9 \%$ de todas las personas hospitalizadas, constituyéndose en un problema de salud no solo en personas en estados crónicos sino también en personas en estados agudos. En el Reino Unido, las úlceras por presión se registraron de un $5 \%$ a $32 \%$ de pacientes ingresados en un hospital general (11).

En Brasil el 2\% de las úlceras por presión son muy severas y pueden comprometer la vida del enfermo. Se calcula que su incidencia en la población general es del $1.7 \%$ entre los 55 y 69 años y del $3.3 \%$ entre los 70 y 75 años. El 60\% aproximadamente se desarrollan en el hospital y más del $70 \%$ ocurren en mayores de 70 años. Lo más importante es que el $95 \%$ de las úlceras por presión son evitables y por ello es prioritaria la prevención (1).

En el Perú en el Programa de Atención Domiciliaria (PADOMI) - EsSalud reporta que el $53,3 \%$ de ancianos se encontraba en un rango de 80-89 años de edad, de los cuales el 43,4\% presentó un riesgo medio de desarrollar úlceras por presión, teniendo en cuenta las condiciones determinantes que lo incrementan (12). 
Estas cifras son preocupantes, pues las úlceras por presión son prevenibles en un 95\%, por lo que es necesario disponer de estrategias de educación y prevención en programas de capacitación en salud enfocados a las personas cuidadoras, siendo en la mayoría de los casos uno o varios miembros de la familia, para que ellas obtengan herramientas que beneficien al usuario o usuaria (8).

La familia es la unidad de Salud que incluye en el núcleo familiar a todas las personas que viven bajo una misma unidad residencial entre los cuales existen lazos de dependencia, obligaciones recíprocas y que, por lo general, pero no siempre, están ligados por lazos de parentesco (1).

La familia asume un rol protagónico como agente de cuidado de los adultos mayores, de manera particular en la prevención de úlceras por presión cuando el paciente tiene una permanencia prolongada en cama.

Se refiere que el 55,6\% de los ancianos son cuidados por sus hijos y otros familiares, presentando en ambos casos un riesgo medio para desarrollar úlceras por presión. Situación que alerta a los profesionales de salud $y$ principalmente a las enfermeras, quienes deben implementar programas de educación continua y entrenamiento supervisando al familiar $\mathrm{y} / \mathrm{o}$ cuidador, para prevenir el desarrollo de úlceras por presión en esta población. En España, el cuidado principal de los ancianos residentes en domicilio recae el cuidado, en el $46 \%$ de los casos, en los hijos (12).

En el Perú se reporta que el 35\% de los ancianos de 60 años a más viven con los hijos. El agente de cuidado o proveedor de cuidados tiene que aprender a participar en el cuidado de su paciente y hacerlo de modo continuo, lo que constituye una característica humana fundamental. Los requisitos infaltables, para realizar dicho cuidado, son el aprendizaje y el uso de conocimientos para llevar a cabo las acciones que faciliten la prevención eficaz y oportuna de las úlceras por presión (4).

En ese sentido, Gallo, refiere que "lamentablemente en nuestro medio no existen programas preventivo-promocionales a favor del adulto mayor, falta mayor incentivo en las instituciones de formación de profesionales de salud para interesarse en el paciente geriátrico, así como de un adiestramiento adecuado para su atención en los diferentes niveles de salud".

Hoy conocemos que las úlceras por presión es un problema común en el cuidado de los pacientes con enfermedades crónicas, sobre todo en ancianos con movilidad limitada, con importante morbimortalidad y elevada repercusión económica y social y sabemos que: "El cuidado de las úlceras es un problema cotidiano, que incluye, medidas de prevención eficaces, un diagnóstico preciso y un tratamiento adecuado".

Las úlceras por presión provocan una prolongación de la estancia hospitalaria y un aumento de los costes sanitarios difícil de asumir sin cuestionar; la inserción de la atención domiciliaria posterior al egreso hospitalario representa una alternativa viable al combinar la atención en salud con esquemas de monitoreo y educación a pacientes y cuidadores, que permiten proporcionar cuidados a largo plazo en el hogar con la atención de equipo médico.

En los últimos tiempos se ha visto que los sistemas nacionales de salud han experimentado un cambio profundo, incremento de los costes de la atención a consecuencia del aumento del número de personas con dependencia sociosanitaria. La elevación de los costes deriva, generalmente, del consumo de recursos hospitalarios o de instituciones que prestan asistencia a ancianos especialmente debilitados.

En este contexto, Perú atiende a una población con diversidad socioeconómica, lo que requiere decisiones eficaces a los problemas de salud complejos, en el cual la atención domiciliaria es un recurso por el que se provee asistencia sanitaria continuada para la resolución en el domicilio de los problemas de salud a las personas que por su situación no pueden desplazarse a un centro sanitario, entendiendo que el nivel de complejidad de los problemas a resolver no requiere la hospitalización ni la institucionalización del sujeto (13).

La atención domiciliaria (AD) es presentada como una de las maneras de atender las demandas que emergen de los cambios en el proceso saludmolestia. La Organización Mundial de la Salud (OMS), en el inicio del siglo XXI, justificó la asistencia domiciliaria como una necesidad frente a los cuidados a largo plazo en las molestias crónicas, que vienen aumentando con la transición demográfica y epidemiológica vivida en los últimos años (14).

Históricamente, el cuidado en el domicilio surgió entre las enfermeras norteamericanas, al final del siglo XIX a través de la Visiting Nursing Association (2) Fue, originalmente, llamado de home care, significando lar y cuidado, respectivamente, siendo en el idioma portugués traducido como cuidados realizados en el domicilio (14).

La atención domiciliaria se asoció con el sexo femenino, el estado socioeconómico bajo, enfermedades crónicas, discapacidad; es percibida por los familiares como deshospitalización. Los 
familiares se sienten frustrados cuando el paciente recibe el alta del hospital antes de estar curado, ya que relacionan el alta a la cura, revelando que no aceptan salir del hospital en cuanto que los pacientes son incapaces de cuidar de sí mismos. En este sentido, los cuidados domiciliarios son vistos como un medio de disminuir la carga de trabajo de los hospitales, destacando la importancia de la preparación previa de los cuidadores domiciliarios, para que puedan conferir cuidado adecuado al paciente en casa (12).

La atención a domicilio en sus inicios era la única forma de atención médica, reservada a algunos privilegiados que se la podían permitir. Por su lado los hospitales estaban destinados para cuidados a los indigentes o a quienes no disponían de una familia que les pudiera atender durante la enfermedad o muerte.

El desarrollo de las técnicas diagnósticas y de tratamiento ha favorecido que los hospitales se conviertan en el centro de atención de los problemas de salud, dejando atrás la atención en domicilio. Sin embargo en los últimos años se ha despertado nuevamente el interés por la atención en domicilio, forzados por motivos económicos, y por el aumento de las enfermedades crónicas (14).

La atención a domicilio, es un recurso que permite proveer asistencia sanitaria para la resolución en el domicilio del paciente, problemas de salud, que por su situación de incapacidad física no puede desplazarse a un centro de salud, entendiendo que el nivel de complejidad de los problemas de salud a resolver, no requiere la hospitalización del paciente, la realiza el equipo de atención primaria, y tiene características que la definen y la diferencian de la hospitalización en domicilio (15).

La atención a domicilio "es un conjunto de actividades que surgen del contexto de un planificación previa, desarrollado por profesionales de un equipo de salud y que tienen como objetivo proporcionar atención de salud mediante actividades que incluyen contenidos de promoción, protección, curación y rehabilitación, dentro de un marco de plena corresponsabilización del usuario y/o familia con los profesionales de la salud, en el domicilio de aquellas personas, que debido a sus estado de salud o a otros criterios no pueden desplazarse al centro de salud" (14).

Según la Organización Mundial de la Salud (OMS), la atención domiciliaria es aquella modalidad de asistencia programada que lleva al domicilio del paciente, los cuidados y atenciones biopsicosociales y espirituales.
No debemos olvidar que el domicilio es el hábitat natural de las personas, en el se ubica el núcleo familiar, es el sitio de referencia del paciente y llega a constituir la parte más importante de su historia personal. El domicilio se constituye en el espacio de calidez, intimidad, de acompañamiento, de comprensión permanente e incondicional, aspecto que cobra mayor importancia en el caso de pacientes con enfermedades crónicas y/o terminales, cuya carga emocional y espiritual se intensifica en los últimos momentos de su vida (16).

La atención a domicilio se constituye en una opción por parte del usuario dependiente de recibir atención continuada, integral y multidisciplinaria, agrupada en un conjunto de servicios que se prestan desde los equipos de salud, mediante funciones y tareas sanitarias $\mathrm{y}$ sociales.

La atención a domicilio tiene como particularidad principal que la consulta se realiza en el domicilio del paciente, fuera del entorno sanitario, por lo que el material complementario para realizar el diagnóstico es limitado.

La atención a domicilio se constituye en un servicio planificado, concertado con el usuario que puede surgir por iniciativa de algún miembro del equipo de salud (atención a domicilio programada), o por requerimiento del paciente y/o algún miembro de su familia (atención a domicilio solicitada) (15).

La atención a domicilio tiene como principio acercar los recursos socio sanitarios de la comunidad y proporcionar asistencia integral en el medio cercano a la persona. En la Conferencia Internacional de Atención Domiciliaria, celebrada en Lombardía en 1995 se establecieron varios principios básicos (14).

El objetivo de la atención a domicilio, es la atención sanitaria directa, tanto de procesos crónicos, crónicos reagudizados, así como agudos. Es también útil para prevenir y detectar otros problemas de salud, fomentar el autocuidado, acercando los servicios sanitarios a la población, y detectar y abordar los problemas sociales que afectan al paciente domiciliario y su familia.

La atención a domicilio se constituye en un instrumento para conocer el medio en el que viven las personas y su grupo familiar, valorar su capacidad de autonomía y su nivel de dependencia, y proporcionar apoyo en el ámbito del cuidado y la asistencia sanitaria (15).

El aumento de la población de adultos mayores y la dependencia, son dos factores que más influyen en la importancia de esta actividad. 
La atención domiciliaria tiene el potencial de aumentar el acceso a los servicios para las personas mayores con discapacidad, para humanizar la atención y fortalecer el vínculo de los equipos de salud con la población. Por otra parte, también reduce las complicaciones clínicas de multimorbilidad relacionados con los cambios morfológicos en la piel en la persona mayor, que pueden tener un claro correlato clínico, favoreciendo una mayor predisposición a que fuerzas mecánicas, por presión, roce o cizalladura, de escasa envergadura o presentes un breve tiempo, o la presencia de un exceso de humedad en la zona expuesta, puedan generar lesiones definitivas y de severo alcance.

El uso frecuente de los servicios hospitalarios por las personas mayores los expone a consecuencias adversas de la hospitalización, muchas de las cuales no están relacionadas a la enfermedad de ingreso sino a efectos adversos de los tratamientos médicos y la práctica hospitalaria. A pesar de que los hospitales modernos tienen lo último en tecnología, muchas prácticas del cuidado del paciente son arcaicas basadas más en tradición y hábitos que en ciencia y que pueden traer consecuencias no deseadas para las personas mayores (17).

Una consecuencia frecuente y potencialmente evitable de la práctica hospitalaria, son las úlceras por presión siendo responsables de agravar el pronóstico de la enfermedad, de disminuir la esperanza de vida y de deteriorar la calidad de vida de las personas que las padecen y también de sus cuidadores.

El propósito principal del presente trabajo, es determinar la efectividad del programa de atención domiciliaria en el cuidado de ulceras por presión y de esta manera resaltar el cumplimento de las intervenciones de enfermería, con el fin de brindar una mejor calidad de vida a los pacientes.

\section{MATERIALES Y MÉTODOS}

Las revisiones sistemáticas son un diseño de investigación observacional y retrospectivo, que sintetiza los resultados de múltiples investigaciones primarias. Son parte esencial de la enfermería basada en la evidencia por su rigurosa metodología, identificando los estudios relevantes para responder preguntas específicas de la práctica clínica. La población constituida por la revisión bibliográfica de 10 artículos científicos publicados e indizados en las bases de datos científicos y que responden a artículos publicados en idioma español, inglés y portugués. La recolección de datos se realizó a través de la revisión bibliográfica de artículos de investigaciones tanto nacionales como internacionales que tuvieron como tema principal la efectividad del programa de atención domiciliaria en el cuidado de ulceras por presión; de todos los artículos que se encontraron, se incluyeron los más importantes según nivel de evidencia y se excluyeron los menos relevantes. Se estableció la búsqueda siempre y cuando se tuvo acceso al texto completo del artículo científico. El algoritmo de búsqueda sistemática de evidencias fue el siguiente:

"Efectividad AND programa de atención domiciliaria AND ulceras por presión. Efectividad AND programa de atención domiciliaria AND enfermería Programa de Atención domiciliaria AND ulceras por presión" Las bases de datos consultadas fueron Pubmed, Medline, Elservier, Lilacs, Lipecs, Scielo, Science Direct.

El análisis de la revisión sistemática está conformado por la elaboración de una tabla de resumen (Tabla 2) con los datos principales de cada uno de los artículos seleccionados, evaluando cada uno de los artículos para una comparación de los puntos o características en las cuales concuerda y los puntos en los que existe discrepancia entre artículos nacionales e internacionales. Además, de acuerdo a criterios técnicos pre-establecidos, se realizó una evaluación crítica e intensiva de cada artículo, a partir de ello, se determinó la calidad de la evidencia y la fuerza de recomendación para cada artículo. La evaluación critica de los artículos científicos revisados, está de acuerdo a las normas técnicas de la bioética en la investigación verificando que cada uno de ellos haya dado cumplimiento a los principios éticos en su ejecución.

\section{RESULTADOS}

Los resultados del presente estudio se resumen en la Tabla 1.

\section{DISCUSIÓN}

En la búsqueda de datos se examinó la efectividad del programa de atención domiciliaria en el cuidado de úlceras por presión. Se encontraron diversos artículos científicos y para ello se utilizó la base de datos Pubmed, Medline, Elservier, Lilacs, Lipecs, Scielo, Science Direct. 


\begin{tabular}{|c|c|c|c|c|}
\hline Diseño de estudio / Titulo & Conclusiones & $\begin{array}{l}\text { Calidad de } \\
\text { evidencias (según } \\
\text { sistema Grade) }\end{array}$ & $\begin{array}{l}\text { Fuerza de } \\
\text { recomendación }\end{array}$ & País \\
\hline $\begin{array}{l}\text { Metaanálisis } \\
\text { Caracterización de salud, } \\
\text { dependencia, inmovilidad y riesgo } \\
\text { de úlceras por presión de } \\
\text { enfermos ingresados al programa } \\
\text { de atención domiciliaria }\end{array}$ & $\begin{array}{l}\text { La atención domiciliaria como actividad de } \\
\text { nivel primario es efectiva, ello por las } \\
\text { necesidades que surgen de los enfermos que } \\
\text { no pueden asistir por sus medios a los } \\
\text { centros asistenciales, por la dependencia } \\
\text { asociada al envejecimiento, aumento de las } \\
\text { enfermedades cardiovasculares, accidentes, } \\
\text { violencia y cáncer. }\end{array}$ & Alta & Fuerte & Chile \\
\hline $\begin{array}{l}\text { Revisión sistemática } \\
\text { Enunciados sobre la atención } \\
\text { domiciliaria en el panorama } \\
\text { mundial: revisión narrativa. }\end{array}$ & $\begin{array}{l}\text { La atención domiciliaria es efectiva ya que } \\
\text { contribuye para promover bienestar y } \\
\text { satisfacción de las necesidades de los } \\
\text { pacientes crónicos y de los moribundos. }\end{array}$ & Alta & Fuerte & España \\
\hline $\begin{array}{l}\text { Revisión sistemática } \\
\text { Cuidados de enfermería en un } \\
\text { paciente con herida crónica en } \\
\text { atención domiciliaria }\end{array}$ & $\begin{array}{l}\text { La atención domiciliaria es efectiva ya que } \\
\text { permitió minimizar las complicaciones y } \\
\text { mejorar en el autocuidado de los pacientes. }\end{array}$ & Alta & Fuerte & Argentina \\
\hline $\begin{array}{l}\text { Revisión sistemática } \\
\text { Efectividad de la atención } \\
\text { domiciliar de enfermería en el } \\
\text { mejoramiento del autocuidado de } \\
\text { personas adultas mayores. }\end{array}$ & $\begin{array}{l}\text { Es efectiva la atención domiciliaria ya que } \\
\text { las personas adultas mayores que reciben } \\
\text { este servicio del profesional de enfermería } \\
\text { tienen un enfoque de promoción de la salud } \\
\text { y prevención de la enfermedad, mejorando } \\
\text { su autocuidado. }\end{array}$ & Alta & Fuerte & Costa Rica \\
\hline $\begin{array}{l}\text { Estudio clínico experimental } \\
\text { Impacto de la visita domiciliaria } \\
\text { en la capacidad funcional de } \\
\text { pacientes con úlceras venosas. }\end{array}$ & $\begin{array}{l}\text { La visita domiciliaria es efectiva ya que las } \\
\text { indicaciones en el marco domiciliario } \\
\text { resultaron beneficiosas para los integrantes } \\
\text { del grupo caso. }\end{array}$ & Alta & Fuerte & Brasil \\
\hline $\begin{array}{l}\text { Cohorte Prospectiva } \\
\text { Impacto de un programa de } \\
\text { atención domiciliaria al enfermo } \\
\text { crónico en ancianos: calidad de } \\
\text { vida y reingresos hospitalarios. }\end{array}$ & $\begin{array}{l}\text { El programa de atención domiciliaria } \\
\text { mejoró la dimensión psicosocial de calidad } \\
\text { de vida de los pacientes crónicos. }\end{array}$ & Moderada & Débil & México \\
\hline $\begin{array}{l}\text { Cuantitativo- transversal } \\
\text { Úlcera por presión después del } \\
\text { alta hospitalaria y los cuidados a } \\
\text { domicilio. }\end{array}$ & $\begin{array}{l}\text { Atención domiciliaria es efectiva como } \\
\text { parte de la Estrategia de Salud de la Familia, } \\
\text { contribuye a reforzar la importancia de los } \\
\text { cuidados de los pacientes, brindando una } \\
\text { atención integral y humanizada. }\end{array}$ & Baja & Débil & Brasil \\
\hline $\begin{array}{l}\text { Observacional -Descriptivo. } \\
\text { Utilización de recursos } \\
\text { sociosanitarios y características } \\
\text { del cuidado informal de los } \\
\text { pacientes inmovilizados en } \\
\text { atención domiciliaria. }\end{array}$ & $\begin{array}{l}\text { Es efectiva y elevada la satisfacción con la } \\
\text { atención domiciliaria. Los pacientes hacen } \\
\text { un uso reducido de recursos sociosanitarios. }\end{array}$ & Baja & Débil & España \\
\hline $\begin{array}{l}\text { Cuantitativo- descriptivo } \\
\text { Úlceras Por Presión En Pacientes } \\
\text { En Cuidados Paliativos } \\
\text { Domiciliarios: Prevalencia Y } \\
\text { Características. }\end{array}$ & $\begin{array}{l}\text { Los equipos domiciliarios son efectivos ya } \\
\text { que permiten detectar las ulceras por presión } \\
\text { e invertir en atención preventiva y } \\
\text { tratamiento eficaces para proporcionar más } \\
\text { comodidad y calidad de vida a esta } \\
\text { población. }\end{array}$ & Baja & Débil & Brasil \\
\hline $\begin{array}{l}\text { Cuantitativo- descriptivo } \\
\text { Caracterización de salud, } \\
\text { dependencia, inmovilidad y riesgo } \\
\text { de úlceras por presión de } \\
\text { enfermos ingresados al programa } \\
\text { de atención domiciliaria. }\end{array}$ & $\begin{array}{l}\text { El programa de atención domicilia es } \\
\text { efectiva ya que, con respecto a la integridad } \\
\text { de la piel, se encontraron baja incidencia de } \\
\text { UPP y éstas en estadios superficiales. }\end{array}$ & Moderada & Débil & Chile \\
\hline
\end{tabular}

Tabla 1. Estudios incluidos en la revisión sistemática

Según los resultados obtenidos de la revisión sistemática, muestran que, del total de 10 artículos revisados, el $100 \%(n=10 / 10)$ afirma que los cuidados en ulceras por presión son efectivos en el programa de atención domiciliaria. Los artículos especifican que las úlceras por presión constituyen un importante problema de salud pública, por sus repercusiones en el nivel de salud de quienes las padecen, la calidad de vida de los usuarios y su entorno y el consumo de recursos para el sistema de salud.

Oliveira; Kruse; Silva y Echevarría (18) refieren que la atención domiciliaria es efectiva y contribuye para promover bienestar y satisfacción 
de las necesidades de los pacientes crónicos y de los moribundos. Refieren que la atención domiciliaria está relacionada con la oferta de cuidados paliativos, de este modo, se afirma que el domicilio es un lugar para estar el enfermo cuando no hay posibilidades de cura. También refieren que los cuidados domiciliarios significan proximidad con el paciente en situación de terminalidad, por mantenerlo dentro de la dinámica familiar y vida social. Los cuidados domiciliarios, junto a sus familiares, es un privilegio, destacando el poder tener acceso a sus pertenencias, preservando su independencia. También los cuidados y las informaciones recibidas por el personal de salud son adecuados y proporcionaron seguridad. En cuanto a la seguridad en la atención domiciliaria está relacionada a la confianza en el sistema de salud. Los familiares confían en el sistema de salud, principalmente al percibir sinceridad en el cuidado de los profesionales y sentirse confortables para hacer preguntas y obtener informaciones sobre el tratamiento.

Cuminao (19) refiere que la atención domiciliaria es efectiva ya que permitió minimizar las complicaciones y mejorar en el autocuidado de los pacientes. A su vez nos dice que mediante las programaciones de las curaciones domiciliarias se pudo lograr minimizar las complicaciones de la herida, en un plazo medianamente oportuno, logrando una buena aceptación y colaboración del paciente sobre el autocuidado.

A su vez Vargas (20) coincide con Oliveira; Kruse; Silva y Echevarría, que es efectiva la atención domiciliaria, basada en el cuidado del paciente, guiada por los principios de promoción de la salud, el empoderamiento, la asociación y centrada en la familia como seguridad de atención. Discrepando en el tipo de pacientes ya que indica que las personas adultas mayores se deben tratar en el domicilio, a su vez reducen los resultados adversos para la salud, tales como caídas, eventos agudos y las hospitalizaciones. Vargas incrementa que es importante la función de enfermería ya que tiene un enfoque de promoción de la salud y prevención de la enfermedad, mejorando su autocuidado. Refiere que las personas adultas mayores que reciben atención domiciliaria del profesional de enfermería desde un enfoque de la promoción de la salud y prevención de la enfermedad mejoran su autocuidado. En relación con lo mencionado, se indica que las personas que reciben una intervención basada en un enfoque crítico de la reflexión tienen una mayor independencia y capacidad percibida para gestionar su propia salud.
Oscar y Sara (21), nos dicen que la atención domiciliaria como actividad de nivel primario es efectiva, ello por las necesidades que surgen de los enfermos que no pueden asistir por sus medios a los centros asistenciales, por la dependencia asociada al envejecimiento, aumento de las enfermedades cardiovasculares, accidentes, violencia y cáncer. A su vez refieren que la enfermera en atención domiciliaria asume un rol preponderante en la atención de estos pacientes, y para ello debe contar con marcos teóricos y técnicos adecuados e instrumentos de valoraciones objetivas y validadas que consideren integralmente a los enfermos y su grupo familiar. Ello plantea un gran desafío para la profesión.

Lópes (22) refiere que la visita domiciliaria es efectiva ya que las indicaciones en el marco domiciliario resultan beneficiosas para los pacientes, coincide con Vargas en que se debe de brindar los cuidados a pacientes adultos mayores y crónicos y con Oliveira que es importante el aspecto socio demográfico. Discrepa en cuanto al sexo de los pacientes ya que refiere que son en su mayoría de sexo femenino las que padecen de estas lesiones.

En cuanto a Espinel (23), coincide con lo mencionado anteriormente que el programa de atención domiciliaria mejora y es efectiva la atención de los pacientes, a su vez que es beneficioso para pacientes crónicos, a su vez coincide en la disminución de costos de estancia hospitalaria donde se retrasa clínicamente el reingreso hospitalario y disminuye el tiempo de estancia de los pacientes lo que explica la influencia del programa que permite contener el uso de servicios y diferir esta atención al equipo de visitas domiciliarias. Pero discrepa en el ámbito de acción ya que refiere que mejora la dimensión psicosocial de calidad de vida de los pacientes. La introducción de modelos de atención domiciliaria representa una alternativa para la contención de servicios y para mejorar la red de apoyo en torno al anciano. A su vez la sobrecarga de los cuidadores debe ser una variable a considerar ya que probablemente representa un impacto negativo para el grupo de atención domiciliaria, puesto que el programa seguramente no aporta elementos que disminuyan la sobrecarga de los cuidadores y sí aumenta las responsabilidades del mismo al soportar la estrategia de acción sobre el cuidador.

Encontramos Valeria Moro y Maria Helena Larcher Caliri (24), refieren que la atención domiciliaria es efectiva como parte de la Estrategia de Salud de la Familia, contribuye a reforzar la importancia de los cuidados de los pacientes, brindando una atención integral $\mathrm{y}$ 
humanizada, permitiendo garantizar una articulación adecuada que facilite la continuidad del cuidado y la seguridad del paciente, valora la importancia sociodemográfica, coincidiendo con los autores mencionados, discrepa con respecto a la comunicación sobre su salud, refiere que es necesario mejorar la exactitud de las indicaciones para atención domiciliaria durante la hospitalización, y corresponde crear mecanismos de comunicación entre los servicios de salud, para garantizar una articulación adecuada que permita facilitar la continuidad del cuidado y la seguridad del paciente.

García (25) coincide que es efectiva y elevada la satisfacción con la atención domiciliaria. Los pacientes hacen un uso reducido de recursos sociosanitarios. La Atención Domiciliaria es una modalidad asistencial y el instrumento necesario para que se pueda desarrollar de forma longitudinal los cuidados integrales e integrados de salud en el domicilio a aquellas personas que, por su situación de enfermedad, no puedan desplazarse al centro de salud. Discrepa diciendo que en el programa de atención domiciliaria la mayoría de los cuidadores informales pertenecen al sexo femenino, predominando una edad avanzada y un bajo nivel de estudios. Por otra parte, la duración y la intensidad del cuidado son altas y permanentes en el tiempo, y los principales apoyos provienen de los profesionales, alcanzando un promedio superior a 2 visitas al mes por parte de enfermería, que se incrementa cuando los pacientes presentan UPP o pluripatología. A su vez incorpora que la enfermería de es el principal proveedor y gestor de la Atención Domiciliaria, desempeñando un papel central en la promoción de la salud, la prevención del deterioro funcional y la continuidad de cuidados, atendiendo de forma longitudinal a los pacientes mediante planes de cuidados de enfermería.

De Castro (26) coincide con Oliveira que la atención domiciliaria es efectiva y que se debe brindar a pacientes paliativos o por morir, discrepando en que las personas en cuidados paliativos desarrollan úlceras por presión (UPP) a medida que se acerca la muerte. La prevalencia de UPP es mayor entre los que tenían antecedentes de lesiones previas. Refiere que el desarrollo de UPP es un hecho relevante que ocurre en la población, lo que indica que es primordial incluir medidas preventivas los equipos domiciliarios son efectivos ya que permiten detectar las ulceras por presión e invertir en atención preventiva y tratamiento eficaces para proporcionar más comodidad y calidad de vida a esta población.
Soto (27) coincide diciendo que el programa de atención domicilia es efectiva ya que, con respecto a la integridad de la piel, se encontraron baja incidencia de UPP y éstas en estadios superficiales, a su vez refiere que la realidad unida al cambio epidemiológico, pronostica una situación en la que cada vez se observará un aumento de personas con patologías que en algún momento pueden desencadenar una condición de dependencia, especialmente en adultos y adultos mayores. La dependencia o pérdida de autonomía para realizar las actividades básicas de la vida diaria puede aparecer en cualquier momento de la vida y se considera como uno de los determinantes de calidad de vida, discrepa diciendo que los pacientes que padecen de lesión son postrados, con nivel económico bajo y su cuidador es informal.

La presente revisión contribuye a mejorar la calidad de cuidados ya que las úlceras por presión pueden ser producidas por diversos factores que pueden llegar a generar disminución en la calidad de vida, con el aumento consecuente de costos para los servicios de salud e inclusive el incremento en los índices de morbilidad y mortalidad, por ende, la atención domiciliaria contribuye para promover bienestar y satisfacción de las necesidades de los pacientes crónicos en sus hogares.

\section{CONCLUSIONES}

Los 10 artículos revisados, el 100\% (n=10/10) afirma que los cuidados en úlceras por presión son efectivos en el programa de atención domiciliaria siendo una alternativa a la hospitalización tradicional que conlleva el beneficio adicional de hacer posible y tornar viable el establecimiento de una innovadora y necesaria coalición y alianza estratégica en el campo de la salud pública y la administración sanitaria con calidad y calidez. La familia es la principal proveedora de cuidados de salud. Asimismo, las úlceras por presión constituyen un importante problema de salud pública, por sus repercusiones en el nivel de salud de quienes las padecen, la calidad de vida de los usuarios y su entorno y el consumo de recursos para el sistema de salud. Por otro lado, se identificó que las úlceras por presión son producidas por diversos factores que pueden llegar a generar disminución en la calidad de vida del adulto mayor, con el aumento consecuente de costos para los servicios de salud e inclusive el incremento en los índices de morbilidad y mortalidad, por los factores expuestos es considerado la atención domiciliaria una 
estrategia de atención que es de carácter resolutivo y dirigida especialmente a la persona con corresponsabilidad individual, familiar y social. Finalmente, el ente del cuidado en atención domiciliaria es el personal de enfermería ya que se encarga de brindar cuidados y capacitar al personal, acompañado de un equipo interdisciplinario.

\section{REFERENCIAS BIBLIOGRÁFICAS}

1. Organización Mundial de la Salud. Medicine geriatrics and evidency based aproach. 2009 (citado 14 de Junio 2017). Disponible en: http://www.who.int/patientsafety/information _centre/documents/ps_research_brochure_en. pdf

2. Soldevilla Agreda Javier. Prevalencia de las ulceras por presion.2013 (citado 14 de Junio 2017). Disponible en: https://repositorio.unican.es/xmlui/bitstream/h andle/10902/2603/GonzalezGarciaR.pdf?sequ ence $=1$

3. MINSA. Guía de Implementación del Programa de Familias y Viviendas y Saludables. Dirección general de promoción de la salud 2005. (citado 14 de Junio 2017). Disponible en: http://bvs.minsa.gob.pe/local/promocion/151_ guiafam.pdf

4. Instituto Nacional de Estadística e Informática. Perfil sociodemográfico de la tercera edad. Lima Perú: Censos Nacionales 2007. (citado 14 de Junio 2017). Disponible en: http://www.cepal.org/deype/noticias/noticias/ 7/40337/d2_28Peru_INEI.pdf

5. EsSalud. Mejoramiento de los servicios de atención geriátrica en Lima Metropolitana. (2006). (citado 14 de Junio 2017). Disponible en:

http://revistascientificas.upeu.edu.pe/index.ph p/rc_salud/article/view/199

6. Varela, L. Funcionalidad en el Adulto Mayor previa a su hospitalización a nivel nacional. 2009. (citado 14 de Junio 2017). Disponible en: http://docplayer.es/121044-Funcionalidaden-el-adulto-mayor-previa-a-suhospitalizacion-a-nivel-nacional.html

7. Solís, M. et al. Manual de prevención y cuidados locales de heridas crónicas. 2011. (citado 14 de Junio 2017). Disponible en: https://es.scribd.com/document/177119027/M anual-de-cuidados-y-heridas-cronicas

8. Venegas, G., Castro, J. \& Solano, M. Programa para la prevención de úlceras por presión en personas adultas mayores. 2010. (citado 14 de Junio 2017). Disponible en: http://www.revenf.ucr.ac.cr/ulceras.pdf> ISSN 1409.

9. Montero, F., Arteaga, S. \& Villarreal, S. Úlceras por presión: Prevención y Tratamiento. 2007. (citado 14 de Junio 2017). Disponible en: http://www.redalyc.org/pdf/448/44817860006 .pdf

10. Fleming, K. Ultrasonido terapéutico para las úlceras de decúbito.2008 (citado 14 de Junio 2017). Disponible en: http://www.cochrane.org/reviewa/es/ab00127 5.html>

11.Gallo, K. \& Pachas, CNorton en una población de ancianos de un servicio domiciliario de Lima. 2008. Programa de Prestaciones de Atención Domiciliaria/PADOMI, del Seguro Social de Salud/EsSalud; Lima - Perú. (citado 14 de Junio 2017). Disponible en: http://cybertesis.urp.edu.pe/bitstream/urp/350/ 1/Gutierrez_ja.pdf

12. Ferrer Arnedo, C. Modelos de organización de la atención domiciliaria.2006 (citado 11 diciembre 2016). Disponible en: http://www.jano.es/ficheros/sumarios/1/0/161 2/47/1v0n1612a13089894pdf001.pdf.

13. Dieckmann J. Home health administration: an overview. 1997 (citado 11 diciembre 2016). Disponible en: http://scielo.isciii.es/pdf/eg/v14n39/revision4. pdf

14. Esteban JM, Ramírez D, Sánchez F. Guía de Buena Práctica Clínica en Atención Primaria. Ed IM \&C Organización Médica Colegial de España-Ministerio de Sanidad y Consumo 2005. (citado 11 diciembre 2016). Disponible en:

http://www.scielo.org.bo/scielo.php?script=sc i_nlinks\&ref $=486277 \&$ pid $=$ S 1726 $8958201200020001000003 \& \operatorname{lng}=\mathrm{es}$

15. Arnedo CF. Modelos de la organización de la atención domiciliaria. JANO 2006. (citado 11 diciembre 2016). Disponible en: http://www.scielo.org.bo/scielo.php?script=sc i_nlinks\&ref $=486275 \&$ pid $=$ S 1726 $8958201200020001000001 \& \operatorname{lng}=\mathrm{es}$

16. Aguirreche J, Granadillo B, Lobo O. Abordaje de atención médica domiciliaria al paciente terminal oncológico, barrio San José, Santa Ana de Coro. Médico de Familia 2009. (citado 11 diciembre 2016). Disponible en: http://www.scielo.org.bo/scielo.php?script=sc i_nlinks\&ref=486279\&pid=S 1726$8958201200020001000005 \& \operatorname{lng}=\mathrm{es}$

17. Gamarra Samaniego Pilar. Consecuencias de la hospitalización en el anciano.2008 (citado 
11 diciembre 2016). Disponible en: http://sisbib.unmsm.edu.pe/bvrevistas/spmi/v $14 \mathrm{n} 2 /$ consecuencias.htm

18. Oliveira, Stefanie Griebeler*; Kruse, Maria Henriqueta Luce**; Sartor, Silvia Francine*** y Echevarría-Guanilo, Maria Elena. Enunciados sobre la atención domiciliaria en el panorama mundial: revisión narrativa. 2015 (citado 11 diciembre 2016). Disponible en: http://scielo.isciii.es/scielo.php?script=sci_artt ext\&pid=S1695-61412015003300117

19. Cuminao, G.; Muñoz, A. Cuidados de enfermería en un paciente con herida crónica en atención domiciliaria. 2014. (citado 11 diciembre 2016). Disponible en: http://gehupptucuman.com.ar/gestor/wpcontent/uploads/2014/11/TH003.pdf

20. Vargas Chaves Shirley, Fernández Chaves Jaime José. Efectividad de la atención domiciliar de enfermería en el mejoramiento del autocuidado de personas adultas mayores 2014. (citado 11 diciembre 2016). Disponible en:

http://www.redalyc.org/pdf/448/44832162007 .pdf.

21. Soto Fernández Oscar. Barrios Casas Sara. Caracterización de salud, dependencia, inmovilidad y riesgo de úlceras por presión de enfermos ingresados al programa de atención domiciliaria.2012 (citado 11 diciembre 2016). Disponible en: http://www.scielo.cl/scielo.php?pid=S071795532012000300007\&script=sci_arttext

22.Lopes JoaquimI Fabiana, et al., Impacto de la visita domiciliaria en la capacidad funcional de pacientes con úlceras venosas, 2016 (citado 11 diciembre 2016). Disponible en: scielo.isciii.es/scielo.php?

script=sci_abstract\&pid=S1134928X2008002200005.

23. Claudia Espinel María, et al., Impacto de un programa de atención domiciliaria al enfermo crónico en ancianos: calidad de vida y reingresos hospitalarios, 2011 (citado 11 diciembre 2016). Disponible en: www.scielosp.org/pdf/spm/v53n1/04.pdf.

24. Valeria Moro Jaisa, Helena Larcher Caliri Maria, Úlcera por presión después del alta hospitalaria y los cuidados a domicilio, 2016. (citado 11 diciembre 2016). Disponible en: www.scielo.br/scielo.php?pid=S1414 $81452016000300203 \&$ script=sci...tlng.

25. García Alcaraza Francisco, Delicado Userosb Victoria, Alfaro Espínb Antonia y LópezTorres Hidalgo Jesús. Utilización de recursos sociosanitarios y características del cuidado informal de los pacientes inmovilizados en atención domiciliaria. 2015 (citado 11 diciembre 2016). Disponible en: http://ac.elscdn.com/S0212656714002042/1-s2.0-

S0212656714002042-

main.pdf?_tid=a687de5a-bfd7-11e6-9e0600000aab0f02\&acdnat $=1481484541 \_1$ ea44bc e6e2f644a9fb57ded6620b475

26. De Castro Mendonça Ana Carolina, Queiroz, Dálete Delalibera Corrêa de Faria Mota , ispeps Marcia Bachion Maria, Cássia Mendes Ana Ferreira, Úlceras Por Presión En Pacientes En Cuidados Paliativos Domiciliarios: Prevalencia Y Características. 2014, (citado 11 diciembre 2016). Disponible en: www.scielo.br/scielo.php?pid=S008062342014000200264\&script=sci...tlng.

27. Soto Fernández Oscar. Barrios Casas Sara. Caracterización de salud, dependencia, inmovilidad y riesgo de úlceras por presión de enfermos ingresados al programa de atención domiciliaria.2012 (citado 11 diciembre 2016). Disponible en: http://www.scielo.cl/scielo.php?pid=S0717 95532012000300007\&script=sci_arttext 